\title{
IVUS-guided treatment strategies for definite late and very late stent thrombosis
}

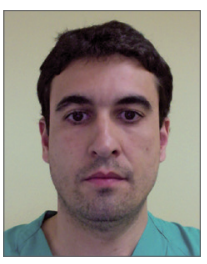

Josep Gomez-Lara ${ }^{1 *}, \mathrm{MD}, \mathrm{PhD}$; Neus Salvatella², MD; Nieves Gonzalo³, MD, PhD;

Felipe Hernández-Hernández ${ }^{4}, \mathrm{MD}$; Eduard Fernandez-Nofrerias 5 , MD;

Angel Sánchez-Recalde ${ }^{6}, \mathrm{MD}$; Teresa Bastante ${ }^{7}, \mathrm{MD}$; Ana Marcano ${ }^{1}$, MD;

Rafael Romaguera ${ }^{1}$, MD; José-Luis Ferreiro ${ }^{1}$, MD; Gerard Roura ${ }^{1}, \mathrm{MD}$; Luis Teruel ${ }^{1}$, MD; Albert Ariza-Solé ${ }^{1}, \mathrm{MD}, \mathrm{PhD}$; Faustino Miranda-Guardiola², MD;

Vera Rodríguez García-Abad ${ }^{3}$, RN; Joan-Antoni Gomez-Hospital ${ }^{1}, \mathrm{MD}, \mathrm{PhD}$; Fernando Alfonso ${ }^{7}, \mathrm{MD}, \mathrm{PhD}$;

Angel Cequier ${ }^{1}, \mathrm{MD}, \mathrm{PhD}$

1. Hospital Universitari de Bellvitge, Institut d'Investigació Biomèdica de Bellvitge (IDIBELL), Universitat de Barcelona, L'Hospitalet de Llobregat, Spain; 2. Hospital del Mar, Grup de Recerca Biomèdica en Malalties del Cor, Hospital del Mar Research Institute (IMIM), Barcelona, Spain; 3. Hospital Clínico San Carlos, Madrid, Spain; 4. Hospital Doce de Octubre, Madrid, Spain; 5. Hospital Germans Trias i Pujol, Badalona, Spain; 6. Hospital La Paz, Madrid, Spain; 7. Hospital La Princesa, Madrid, Spain

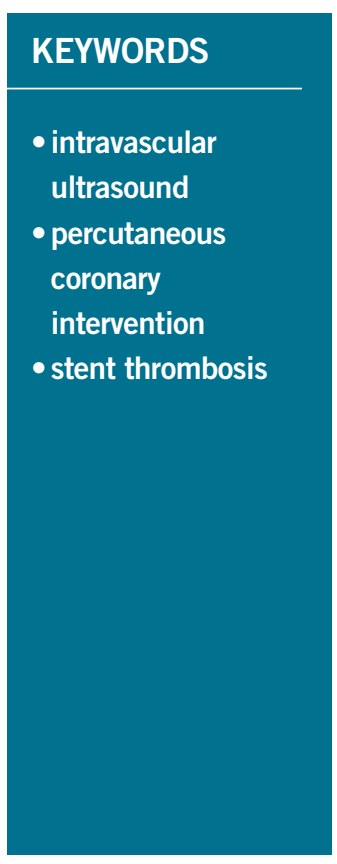

\begin{abstract}
Aims: Our aim was to describe the intravascular ultrasound (IVUS) findings of patients with late stent thrombosis (ST) undergoing percutaneous intervention, and to compare the pre- and post-intervention IVUS findings of patients treated with balloon angioplasty (BA) vs. additional stent implantation (ASI).

Methods and results: A total of 117 patients with late ST imaged with IVUS were included (51.2\% had drug-eluting stent ST). Treatment was left to the operator's discretion: BA was performed in $53.8 \%$ and ASI in 46.2\%. Pre-intervention, incomplete stent apposition (ISA) was observed in 69.8\% vs. $63.0 \%$ $(\mathrm{p}=0.43)$, underexpansion in $33.3 \%$ vs. $18.5 \%(\mathrm{p}=0.07)$ and restenosis in $15.9 \%$ vs. $27.8 \%(\mathrm{p}=0.12)$, respectively. Post-intervention, persistent ISA was observed in $37.2 \%$ vs. $60.9 \%(\mathrm{p}=0.03)$ and malapposition volume decreased by $43.6 \%$ vs. $2.6 \%$ ( $\mathrm{p}=0.03$ ). Persistent underexpansion was observed in $9.3 \%$ vs. $17.4 \%$ $(\mathrm{p}=0.26)$; however, the stent expansion index was largely increased with BA (from 0.75 to 0.88 ) compared to ASI (from 0.80 to 0.82$) ; \mathrm{p}=0.046$. At two years, recurrent ST was observed in one $(1.7 \%)$ vs. four $(7.7 \%)$ patients, respectively; $\mathrm{p}=0.09$.
\end{abstract}

Conclusions: Non-optimal IVUS criteria of stent implantation are often observed in patients with late ST. Treatment of late ST with BA leads to a larger reduction of malapposition and underexpansion with respect to ASI and is associated with favourable outcomes.

*Corresponding author: Department of Interventional Cardiology, Hospital Universitari de Bellvitge, c/ Feixa Llarga sn, 08907 L'Hospitalet de Llobregat, Spain.E-mail: gomezjosep@hotmail.com 


\section{Abbreviations}

ASI additional stent implantation

BA balloon angioplasty

ISA incomplete stent apposition

IVUS intravascular ultrasound

PCI percutaneous coronary intervention

ST stent thrombosis

\section{Introduction}

Definite late and very late stent thrombosis (ST) is defined as any thrombosis observed by angiography or pathology later than one month after stent implantation ${ }^{1}$. The incidence of angiographic late ST is infrequent, but it is associated with poor outcomes at midterm follow-up ${ }^{2-4}$. The cumulative incidence of late ST is around $0.8 \%$ with bare metal stents (BMS), $0.7-1.5 \%$ with first-generation drug-eluting stents (DES), and $0.5-0.7 \%$ with second-generation DES at two to three years of follow-up ${ }^{5-7}$. However, there is no attenuation of the incidence/ year of stent thrombosis with either BMS or DES at very longterm follow-up ${ }^{4,8}$.

Most of the cases with late ST present as acute coronary syndromes undergoing urgent percutaneous coronary intervention $(\mathrm{PCI})^{2,3}$. The most common cause of late and very late DES thrombosis is the observation of incomplete healing and/or inadequate neointimal coverage of the struts ${ }^{9}$. Other causes of late ST are incomplete stent apposition (ISA), stent underexpansion, stent restenosis and the emergence of "vulnerable" neoatherosclerotic plaques within the neointimal tissue, or insufficient antiplatelet treatment ${ }^{10}$.

Although there are few data regarding the best PCI treatment of angiographic late (and very late) ST, current European guidelines recommend the use of thromboaspiration and predilation with non-compliant balloons (Class IIa, level of evidence C) ) $^{1,12}$. Glycoprotein IIb/IIIa inhibitors have also been associated with better procedural outcomes and a lower risk of recurrent thrombosis compared to patients without glycoprotein IIb/IIIa administration $^{6}$. In contrast, additional stent implantation (ASI) has been related to worse cardiac outcomes as compared to patients without stent implantation ${ }^{2,5}$. Most of the clinical outcomes observed in patients treated with ASI were related to cardiac death or recurrent ST at midterm follow-up ${ }^{2}$. Despite this, the rate of stent implantation for late ST treatment is still high, ranging from $40 \%$ to $100 \%$ in different studies ${ }^{2,3,11}$.

Current European guidelines recommend the use of intravascular imaging techniques to detect stent-related mechanical problems (Class IIa, level of evidence C) ${ }^{12}$. However, there are few data on the utility of IVUS as guidance in PCI of patients with late stent thrombosis. The first objective of this study was to describe the IVUS findings of angiographic late and very late ST, and to report the ultrasound results after PCI. The second objective was to compare the IVUS findings and clinical outcomes between patients treated with balloon angioplasty (BA) versus ASI.

\section{Methods POPULATION}

This was a multicentre, observational study that included all patients presenting with angiographic late and very late ST imaged with IVUS in seven Spanish institutions from January 2008 to December 2012. All institutions participating in the study are high-volume centres ( $>500 \mathrm{PCI} /$ year) with considerable experience in the use of IVUS for complex $\mathrm{PCI}^{13}$. A flow chart with all consecutive patients presenting with late ST is shown in Figure 1. All participating institutions followed the recommendations for dual antiplatelet therapy in the current guidelines ${ }^{12}$. In patients receiving a stent (BMS or DES) during PCI for acute coronary syndrome (ACS) or in patients receiving a DES for silent or stable angina, $\mathrm{P}_{2} \mathrm{Y}_{12}$ inhibitor therapy was recommended for at least 12 months plus aspirin. In patients receiving BMS for a non-ACS indication, a $\mathrm{P} 2 \mathrm{Y}_{12}$ inhibitor was recommended for a minimum of one month plus aspirin.

This study was approved by the local ethics committee of all participating institutions and was carried out in accordance with the Declaration of Helsinki. Written informed consent was obtained from all patients. There was no funding source for this study.

\section{PROCEDURE CHARACTERISTICS}

PCI was performed according to standard practice in each participating centre. Unfractionated heparin, low molecular weight heparin or bivalirudin was used for procedural anticoagulation. The use of glycoprotein IIb/IIIa inhibitors was left to the operator's discretion. A loading dose of aspirin ( $\geq 250 \mathrm{mg}$ ) was administered to the patient before PCI. A loading dose of clopidogrel ( $\geq 300 \mathrm{mg})$,

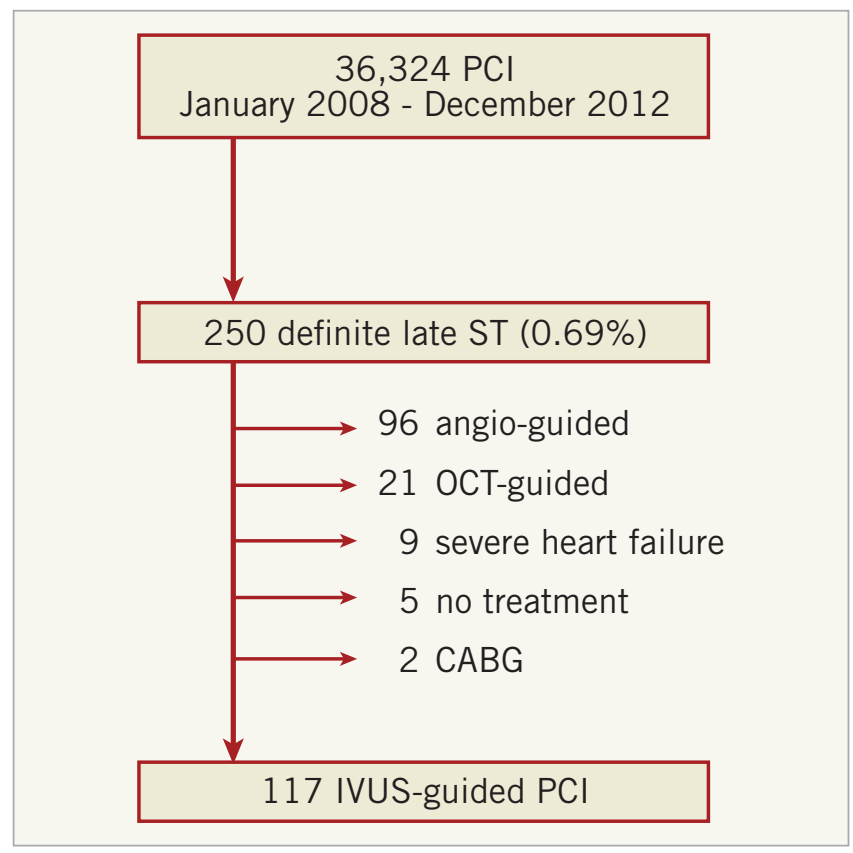

Figure 1. Flow chart of the study. IVUS: intravascular ultrasound; OCT: optical coherence tomography; PCI: percutaneous coronary intervention; ST: stent thrombosis 
prasugrel $(60 \mathrm{mg})$ or ticagrelor $(180 \mathrm{mg})$ was given to the patients according to the physician's preference.

IVUS imaging was performed after restoration of TIMI flow $\geq 2$ with thrombus aspiration or balloon angioplasty at low pressure. These images were used for "pre-intervention analysis". After careful evaluation of IVUS images "on-line", the operator was left to decide the best treatment for the cause of stent thrombosis. All cases were treated with balloon angioplasty. In case of stent implantation, the use of DES or BMS was also left to the operator's discretion. At the end of all coronary interventions, a final IVUS acquisition was performed. These images were used for "post-intervention analysis".

\section{IVUS ACQUISITION}

IVUS acquisition was performed according to standard procedures ${ }^{14}$. After an intracoronary nitroglycerine injection, the catheter probe was advanced distally to the stent segment. Using an automated pullback device, the transducer was withdrawn at a continuous speed of $0.5 \mathrm{~mm} / \mathrm{s}$. One participating institution performed IVUS recording at $1 \mathrm{~mm} / \mathrm{s}$. The image data were stored on DVD for "off-line" analysis. IVUS acquisition was performed with the Atlantis ${ }^{\mathrm{TM}} 40 \mathrm{MHz}$ catheter (Boston Scientific, Marlborough, MA, USA).

\section{"Off-line" IVUS analysis}

IVUS "off-line" analysis was performed by a central core lab (BARCICORE-LAB, Barcelona, Spain) using quantitative intra- vascular ultrasound analysis software, QIvus 3.0 (Medis, Leiden, The Netherlands). The stent segment was defined by the stent edges. According to the pullback speed, the most normal-looking cross-sections within $5 \mathrm{~mm}$ proximal and distal to the stent were defined as the reference lumen areas.

Two independent and blinded analysts were requested to evaluate qualitatively the IVUS pullback and to identify four IVUS findings: late malapposition, aneurysm, stent underexpansion and stent restenosis. Incomplete stent apposition (ISA) was defined as a clear separation of the metallic struts from the vessel wall in the absence of a side branch. Aneurysms were defined as lesions that included all layers of the vessel wall with an external elastic membrane (EEM) and lumen area $>50 \%$ larger than the proximal reference segment ${ }^{14,15}$. Stent underexpansion was defined when the minimal stent area was $\leq 80 \%$ of the reference lumen area. Stent restenosis was defined as all lesions with area stenosis $>50 \%$ due to neointima tissue. Neoatherosclerosis was defined as the presence of clear intra-stent plaque with lipid or calcium echogenic characteristics. Figure 2 shows examples of each IVUS finding.

Quantitative measurements of the lumen, stent and vessel $(=\mathrm{EEM})$ were performed according to the standard procedures ${ }^{14,16,17}$. Stent measurements were obtained by drawing the stent area of the stent with ST. However, after additional stent implantation, stent measurements were obtained by drawing the stent area of the entire stent segment. Neointimal area/volume

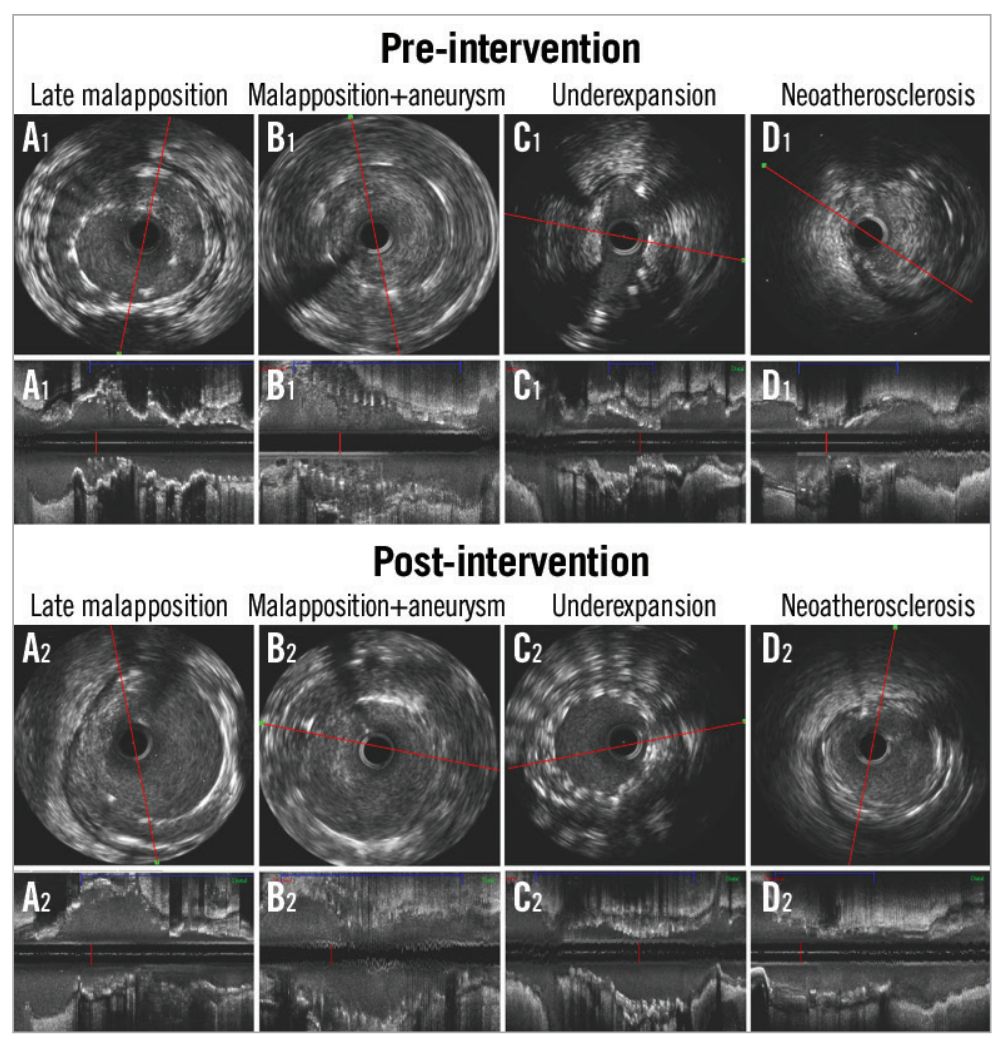

Figure 2. Qualitative IVUS findings pre- and post-intervention. 
were estimated by the following formula: Stent - (Lumen - ISA). Balloon-to-artery ratio was calculated as: largest device-estimated diameter by the manufacturer's device chart at the maximal pressure/reference lumen diameter from the non-stented segment as assessed by IVUS.

\section{CLINICAL FOLLOW-UP}

Clinical follow-up information was obtained from telephone interviews with the patients, their relatives and hospital records. Information regarding the development of major adverse cardiac events, predefined as death, recurrent stent thrombosis, any myocardial infarction and any coronary revascularisation, was collected.

\section{STATISTICAL ANALYSIS}

The Kolmogorov-Smirnov test was used to evaluate the normality assumptions of all continuous variables. Continuous variables were expressed as mean \pm 1 standard deviation. Non-normally distributed variables were expressed as median (interquartile range: IQR). Categorical variables were expressed as counts (\%). Comparisons between pre- and post-intervention were estimated with t-test analysis for paired data. In the case of non-normal distribution, comparisons between pre- and post-intervention were estimated with the non-parametric Wilcoxon test. Comparisons between categorical variables were performed with the chi-square test. Comparisons of clinical outcomes between groups were performed with Cox regression analysis. In order to adjust for baseline differences between the two treatment groups (patients with BA versus ASI) all variables with a $p$-value $\leq 0.10$ were included as covariates in the multivariate model (hypertension, hypercholesterolaemia, body mass index, stent underexpansion prior to any intervention as assessed by IVUS, stent restenosis prior to any intervention as assessed by IVUS, and final TIMI flow <3). Outcomes are presented with hazard ratio (HR; 95\% confidence interval [CI]) and with Kaplan-Meier survival curves. All measures were obtained with SPSS, Version 20.0 (IBM Corp. Armonk, NY, USA).

\section{Results}

\section{BASELINE DEMOGRAPHIC, ANGIOGRAPHIC AND PROCEDURAL CHARACTERISTICS}

A total of 250 patients with late ST were documented during the study period $(0.69 \%$ of all PCIs performed by all institutions participating in the study); 117 lesions in 116 patients with late ST were imaged with IVUS and were included in the present study. Figure 1 shows the flow chart with patient selection. BMS thrombosis was observed in $52(44.4 \%)$ and DES thrombosis in 60 cases $(51.2 \%)$. A total of 63 lesions $(53.8 \%)$ were treated with BA and 54 lesions (46.2\%) with ASI. Baseline demographic and angiographic characteristics are shown in Table 1. At the time of the stent thrombosis, there were no statistical differences between groups except for body mass index (28.4 vs. $\left.26.4 \mathrm{~kg} / \mathrm{m}^{2} ; \mathrm{p}<0.01\right)$. A total of 18 patients were on dual antiplatelet therapy $(16.1 \%$

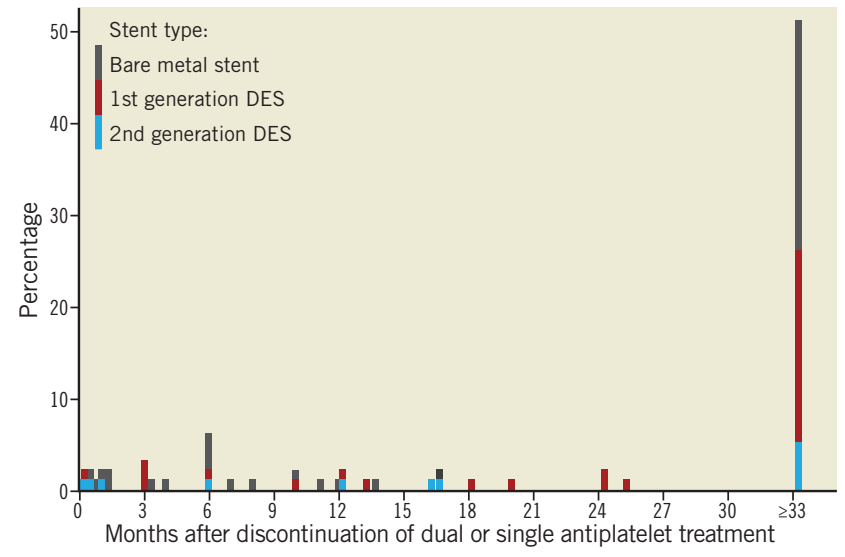

Figure 3. Time to stent thrombosis of patients with dual or single antiplatelet treatment discontinuation.

treated with BA vs. $14.8 \%$ with ASI; $\mathrm{p}=0.90$ ). Figure 3 shows the time to thrombosis after discontinuation of dual or single antiplatelet treatment of patients without dual antiplatelet therapy. The largest balloon and stent diameters $(3.6 \pm 0.5 \mathrm{~mm}$ vs. $3.6 \pm 0.7 \mathrm{~mm}$; $\mathrm{p}=0.88)$ and balloon-to-artery ratios ( $1.30 \pm 0.20$ vs. $1.29 \pm 0.24$; $\mathrm{p}=0.97$ ) were similar between groups. There were five periprocedural complications during PCI: two coronary perforations Ellis type II (one sealed with prolonged balloon inflation and one which required a graft stent), two distal embolisations (one after IVUS imaging and one after stent implantation which caused a temporary no-reflow with ventricular fibrillation), and one coronary haematoma after balloon dilation which required ASI. Appendix Table 1 contains information regarding the baseline clinical, angiographic and procedural characteristics of patients according to stent type.

\section{QUALITATIVE IVUS FINDINGS}

All patients had a pre-intervention IVUS examination. A total of 89 patients $(76 \%)$ also had IVUS imaging at the end of all coronary interventions (post-intervention). Prior to intervention, none of the selected IVUS findings was observed in nine lesions (7.7\%), ISA was observed in $78(66.7 \%)$, underexpansion in 31 (26.5\%), and stent restenosis in $25(21.4 \%)$. There were no differences in the observation of ISA $(69.8 \%$ vs. $63.0 \%$; $\mathrm{p}=0.43)$, underexpansion $(33.3 \%$ vs. $18.5 \% ; \mathrm{p}=0.07)$ or restenosis $(15.9 \%$ vs. $27.8 \%$; $\mathrm{p}=0.12$ ) between lesions treated with BA vs. ASI. Postintervention, persistent ISA was observed in $37.2 \%$ vs. $60.9 \%$ of patients $(\mathrm{p}=0.03)$, persistent underexpansion was observed in $9.3 \%$ vs. $17.4 \%(p=0.26)$, and residual restenosis was not seen in any patient. Table 2 shows the qualitative IVUS findings including all possible combinations of the selected IVUS parameters. Appendix Table 2 contains information regarding the IVUS characteristics of patients according to stent type.

\section{QUANTITATIVE IVUS DATA}

Table 3 summarises the quantitative IVUS analysis. Minimal lumen area showed a similar increase between pre- and 
Table 1. Clinical, angiographic and procedural characteristics.

\begin{tabular}{|c|c|c|c|c|c|}
\hline \multicolumn{2}{|c|}{$n=117$ lesions } & All patients & $\begin{array}{l}\text { No additional stent } \\
\qquad(\mathrm{n}=63)\end{array}$ & $\begin{array}{l}\text { Additional stent } \\
\qquad(n=54)\end{array}$ & $p$-value \\
\hline \multicolumn{6}{|c|}{ Clinical and angiographic characteristics at the time of the stent implantation } \\
\hline \multirow{3}{*}{$\begin{array}{l}\text { Clinical indication } \\
(n=115)\end{array}$} & Silent or stable angina & $21(18.2)$ & $10(16.1)$ & $11(20.8)$ & \multirow{3}{*}{0.10} \\
\hline & Non-STEMI ACS & $34(29.6)$ & $14(22.6)$ & $20(37.7)$ & \\
\hline & STEMI ACS & $60(52.2)$ & $38(61.3)$ & $22(41.5)$ & \\
\hline \multirow[t]{5}{*}{ Culprit artery, n (\%) } & LAD & $66(56.9)$ & $36(57.1)$ & $30(55.6)$ & \multirow{5}{*}{0.72} \\
\hline & LCF & $11(9.5)$ & $6(9.5)$ & $5(9.3)$ & \\
\hline & RCA & $38(32.8)$ & $20(31.7)$ & $18(33.3)$ & \\
\hline & Left main & $1(0.9)$ & 0 & $1(1.9)$ & \\
\hline & Vein graft & $1(0.9)$ & $1(1.6)$ & 0 & \\
\hline \multicolumn{2}{|c|}{ Number of stents, $n \pm S D$} & $1.3 \pm 0.5$ & $1.3 \pm 0.5$ & $1.2 \pm 0.5$ & 0.21 \\
\hline \multicolumn{2}{|c|}{ Total stent length, $\mathrm{mm} \pm \mathrm{SD}$} & $25.9 \pm 13.1$ & $26.2 \pm 11.8$ & $25.5 \pm 14.5$ & 0.79 \\
\hline \multicolumn{2}{|c|}{ Minimal stent diameter, $\mathrm{mm} \pm \mathrm{SD}$} & $3.0 \pm 0.5$ & $3.0 \pm 0.5$ & $3.0 \pm 0.4$ & 0.39 \\
\hline \multirow[t]{7}{*}{ Stent type, n (\%) } & BMS & $52(44.4)$ & $24(38.1)$ & $28(51.9)$ & \multirow{7}{*}{0.19} \\
\hline & SES & $13(11.1)$ & $7(11.1)$ & $6(11.1)$ & \\
\hline & PES & $28(23.9)$ & $18(28.6)$ & $10(18.5)$ & \\
\hline & EES & $13(11.1)$ & $9(14.3)$ & $4(7.4)$ & \\
\hline & ZES & $4(3.4)$ & $1(1.6)$ & $3(5.6)$ & \\
\hline & BES & $2(1.7)$ & 0 & $2(3.7)$ & \\
\hline & Unknown & $5(4.3)$ & $4(6.3)$ & $1(1.9)$ & \\
\hline \multicolumn{6}{|c|}{ Clinical characteristics at the time of the stent thrombosis } \\
\hline \multicolumn{2}{|c|}{ Age (years), median (IQR) } & 61.7 (52.0 to 70.3$)$ & $57.44(50.6$ to 69.4$)$ & 63.4 (52.5 to 71.7$)$ & 0.62 \\
\hline \multicolumn{2}{|c|}{ Men, n (\%) } & $108(92.3)$ & $57(90.5)$ & $51(94.4)$ & 0.42 \\
\hline \multicolumn{2}{|c|}{ Hypertension, n (\%) } & $76(65.0)$ & $36(57.1)$ & $40(74.1)$ & 0.06 \\
\hline \multicolumn{2}{|c|}{ Hypercholesterolaemia, n (\%) } & $87(74.4)$ & $43(68.3)$ & $44(81.5)$ & 0.10 \\
\hline \multicolumn{2}{|c|}{ Diabetes mellitus, $\mathrm{n}(\%)$} & $43(36.8)$ & $21(33.3)$ & $22(40.7)$ & 0.41 \\
\hline \multirow{3}{*}{$\begin{array}{l}\text { Smoking status, } n \\
(\%)\end{array}$} & Never & $27(23.1)$ & $14(22.2)$ & $13(24.1)$ & \multirow[t]{3}{*}{0.24} \\
\hline & Ex-smoker & $44(37.6)$ & $20(31.7)$ & $24(44.4)$ & \\
\hline & Current smoker & $46(39.3)$ & $29(46.0)$ & $17(31.5)$ & \\
\hline \multicolumn{2}{|c|}{ Body mass index, median (IQR) } & 27.7 (24.6 to 31.1 ) & 28.4 (26.0 to 32.0 ) & 26.4 (24.2 to 28.8 ) & 0.01 \\
\hline \multicolumn{2}{|c|}{ Ejection fraction (\%), median (IQR) } & 55.0 (45.0 to 60.0$)$ & 51.5 (45.0 to 60.0 ) & 55.0 (45.0 to 60.0$)$ & 0.86 \\
\hline \multicolumn{2}{|c|}{ Creatinine clearance $(\mathrm{ml} / \mathrm{min})$, median (IQR) } & 80.8 (58.0 to 97.2$)$ & $88.9(69.3$ to 100.1$)$ & $67.8(50.8$ to 87.9$)$ & 0.30 \\
\hline \multirow{3}{*}{$\begin{array}{l}\text { Current antiplatelet } \\
\text { treatment, } \mathrm{n}(\%)\end{array}$} & None & $12(10.3)$ & $7(11.3)$ & $5(9.3)$ & \\
\hline & Monotherapy & $86(74.1)$ & $45(72.6)$ & $41(75.9)$ & 0.90 \\
\hline & Dual antiplatelet treatment & $18(15.5)$ & $10(16.1)$ & $8(14.8)$ & \\
\hline Time to thrombosis & ars), median (IQR) & $3.6(1.0$ to 6.2$)$ & $3.5(0.9$ to 6.2$)$ & $3.6(1.2$ to 6.5$)$ & 0.94 \\
\hline Clinical & Non-STEMI ACS & $18(15.4)$ & $7(11.1)$ & $11(20.4)$ & 028 \\
\hline presentation, n (\%) & STEMI ACS & $99(84.6)$ & $56(88.9)$ & $43(79.6)$ & 0.28 \\
\hline Killip class, n (\%) & 1 & $87(74.4)$ & $46(73.0)$ & $41(75.9)$ & 0.55 \\
\hline & II & $19(16.2)$ & $9(14.3)$ & $10(18.5)$ & \\
\hline & III & $6(5.1)$ & $4(6.3)$ & $2(3.7)$ & \\
\hline & IV & $5(4.3)$ & $4(6.3)$ & $1(1.9)$ & \\
\hline Angiographic and & cedural characteristics at & time of the stent thro & & & \\
\hline TIMI flow pre- & 0 & $85(72.6)$ & $43(68.3)$ & $42(77.8)$ & \\
\hline intervention, n (\%) & 1 & $6(5.1)$ & $2(3.2)$ & $4(7.4)$ & \\
\hline & 2 & $8(6.8)$ & $4(6.3)$ & $4(7.4)$ & 0.13 \\
\hline & 3 & $18(15.4)$ & $14(22.2)$ & $4(7.4)$ & \\
\hline
\end{tabular}


Table 1. Clinical, angiographic and procedural characteristics. (cont'd)

\begin{tabular}{|c|c|c|c|c|c|}
\hline \multicolumn{2}{|c|}{$\mathrm{n}=117$ lesions } & All patients & $\begin{array}{l}\text { No additional stent } \\
\qquad(n=63)\end{array}$ & $\begin{array}{l}\text { Additional stent } \\
\qquad(n=54)\end{array}$ & $p$-value \\
\hline \multicolumn{6}{|c|}{ Angiographic and procedural characteristics at the time of the stent thrombosis } \\
\hline \multicolumn{2}{|c|}{ Thromboaspiration, $\mathrm{n}(\%)$} & $92(78.6)$ & $49(77.8)$ & $43(79.6)$ & 0.81 \\
\hline \multicolumn{2}{|c|}{ Glycoprotein IIb/IIla inhibitors, n (\%) } & $75(64.1)$ & $43(68.3)$ & $32(59.3)$ & 0.31 \\
\hline \multicolumn{2}{|c|}{ Number of additional stents, $\mathrm{n} \pm \mathrm{SD}$} & $1.3 \pm 0.7$ & - & $1.3 \pm 0.7$ & NA \\
\hline \multirow{2}{*}{$\begin{array}{l}\text { Additional stent type, } \\
\text { n (\%) }\end{array}$} & BMS & - & - & $27(50.0)$ & \multirow{2}{*}{ NA } \\
\hline & DES & - & - & $27(50.0)$ & \\
\hline \multicolumn{2}{|c|}{ Post-dilatation, n (\%) } & - & - & $20(37.0)$ & NA \\
\hline \multicolumn{2}{|c|}{ Largest device nominal diameter, $\mathrm{mm} \pm \mathrm{SD}$} & $3.6 \pm 0.6$ & $3.6 \pm 0.5$ & $3.6 \pm 0.7$ & 0.88 \\
\hline \multicolumn{2}{|c|}{ Largest device estimated diameter, $\mathrm{mm} \pm \mathrm{SD}$} & $3.8 \pm 0.6$ & $3.8 \pm 0.6$ & $3.8 \pm 0.7$ & 0.75 \\
\hline \multicolumn{2}{|c|}{ Balloon-to-artery ratio $\pm S D$} & $1.30 \pm 0.22$ & $1.30 \pm 0.20$ & $1.29 \pm 0.24$ & 0.97 \\
\hline \multicolumn{2}{|c|}{ Periprocedural complications, $\mathrm{n}(\%)$} & $5(4.3)$ & $3(4.8)$ & $2(3.7)$ & 0.78 \\
\hline \multirow{4}{*}{$\begin{array}{l}\text { TIMI flow post- } \\
\text { intervention, n (\%) }\end{array}$} & 0 & $1(0.9)$ & 0 & $1(1.8)$ & \multirow{4}{*}{0.09} \\
\hline & 1 & 0 & 0 & 0 & \\
\hline & 2 & $3(2.6)$ & 0 & $3(5.6)$ & \\
\hline & 3 & $113(96.6)$ & 63 (100.0) & $50(92.6)$ & \\
\hline
\end{tabular}

post-intervention in both groups $(31.0 \%$ with BA and $20.7 \%$ with ASI; $p=0.25$ ). Lumen volume showed a lower increase in the group treated with BA than in the group treated with ASI $(16.3 \%$ vs. $65.7 \%$, respectively; $p<0.01)$. However, part of the increase observed in the group treated with ASI was because of the longer segment analysed post-intervention (the stent length increased from $24.1 \mathrm{~mm}$ to $36.0 \mathrm{~mm}$ in the group with ASI; $\mathrm{p}<0.01)$.

Stent areas increased between pre- and post-intervention in both groups, but patients treated without ASI had a larger increase than the group treated with ASI (minimal stent area increased by $29.7 \%$ vs. $12.1 \%$, respectively; $p<0.01$ ). The stent expansion index increased in the group treated with BA from 0.75 to $0.88(\mathrm{p}=0.01)$ but remained unchanged in the group treated with ASI (from 0.80 to $0.82 ; \mathrm{p}=0.92$ ).

In the longitudinal analysis, malapposition length was only reduced between pre- and post-intervention in the group treated with BA (40.5\% decrease) and was unchanged in the group treated with ASI $(1.7 \%$ decrease); $\mathrm{p}<0.01$. Despite the percentage of lumen volume with malapposition being reduced in both groups,

Table 2. Qualitative IVUS findings.

\begin{tabular}{|c|c|c|c|c|}
\hline & All lesions $(n=117)$ & No additional stent $(n=63)$ & Additional stent $(n=54)$ & $p$-value \\
\hline \multicolumn{5}{|c|}{ Qualitative IVUS findings pre-intervention } \\
\hline None, $\mathrm{n}(\%)$ & $9(7.7)$ & $5(7.9)$ & $4(7.4)$ & 0.92 \\
\hline Only malapposition, n (\%) & 49 (41.9) & $26(41.3)$ & $23(42.6)$ & 0.89 \\
\hline Only underexpansion, n (\%) & $15(12.8)$ & $10(15.9)$ & $5(9.3)$ & 0.29 \\
\hline Only restenosis, n (\%) & $12(10.2)$ & $3(4.7)$ & $9(16.6)$ & 0.03 \\
\hline Malapposition+aneurysm & $11(9.4)$ & $6(9.5)$ & $5(9.3)$ & 0.96 \\
\hline Malapposition+underexpansion, n (\%) & $11(9.4)$ & $8(12.7)$ & $3(5.6)$ & 0.19 \\
\hline Underexpansion+restenosis, n (\%) & $3(2.6)$ & $1(1.6)$ & $2(3.7)$ & 0.47 \\
\hline Malapposition+restenosis, n (\%) & $3(2.6)$ & $1(1.6)$ & $2(3.7)$ & 0.47 \\
\hline \multirow[t]{2}{*}{ Other combinations, n (\%) } & $4(3.4)$ & $3(4.8)$ & $1(1.8)$ & 0.39 \\
\hline & All lesions $(n=89)$ & No additional stent $(n=43)$ & Additional stent $(n=46)$ & $p$-value \\
\hline \multicolumn{5}{|c|}{ Qualitative IVUS findings post-intervention } \\
\hline None, n (\%) & $39(43.8)$ & $24(55.8)$ & $15(32.6)$ & 0.03 \\
\hline Only malapposition, n (\%) & $26(29.2)$ & 9 (20.9) & $17(37.0)$ & 0.10 \\
\hline Only underexpansion, n (\%) & $6(6.7)$ & $3(7.0)$ & $3(6.5)$ & 0.93 \\
\hline Malapposition+aneurysm & $12(13.5)$ & $6(14.0)$ & $6(13.0)$ & 0.90 \\
\hline Malapposition+underexpansion, n (\%) & $6(6.7)$ & $1(2.3)$ & 5 (10.9) & 0.11 \\
\hline
\end{tabular}


Table 3. Quantitative IVUS findings.

\begin{tabular}{|c|c|c|c|c|c|c|c|c|c|c|}
\hline \multirow[b]{2}{*}{$\mathrm{n}=117$ lesions } & \multicolumn{4}{|c|}{ No additional stent $(n=63)$} & \multicolumn{4}{|c|}{ Additional stent $(n=54)$} & \multirow[b]{2}{*}{$p$-value ${ }^{\infty}$} & \multirow[b]{2}{*}{$p$-value } \\
\hline & $\begin{array}{c}\text { Pre- } \\
\text { intervention }\end{array}$ & $\begin{array}{l}\text { Post- } \\
\text { intervention }\end{array}$ & $\begin{array}{c}\text { Absolute } \\
\text { (relative } \\
\text { difference } \\
\% \text { ) }\end{array}$ & $p$-value & $\begin{array}{c}\text { Pre- } \\
\text { intervention }\end{array}$ & $\begin{array}{l}\text { Post- } \\
\text { intervention }\end{array}$ & \begin{tabular}{|c|} 
Absolute \\
(relative \\
difference \\
$\%$ ) \\
\end{tabular} & $\boldsymbol{p}$-value & & \\
\hline \multicolumn{11}{|l|}{ Diameters (mm \pm SD) } \\
\hline Stent length & 22.7 (16.7 to 30.6$)$ & 22.3 (17.7 to 28.9 ) & $-1.0(-3.7)$ & 0.14 & 22.0 (18.0 to 29.3$)$ & 29.8 (25.6 to 40.5$)$ & $11.9(49.4)$ & $<0.01$ & 0.25 & $<0.01$ \\
\hline Malapposition length* & $13.1(5.5$ to 18.0$)$ & 3.0 (0 to 15.4) & $-6.0(-40.5)$ & $<0.01$ & 15.1 (10.9 to 22.4) & $18.6(6.0$ to 27.0$)$ & $-0.3(-1.7)$ & 0.90 & 0.42 & $<0.01$ \\
\hline \multicolumn{11}{|l|}{ Areas $\left(\mathrm{mm}^{2} \pm \mathrm{SD}\right)$} \\
\hline Ref lumen area & $9.0 \pm 3.6$ & $10.0 \pm 4.3$ & $0.7(10.8)$ & 0.12 & $8.6 \pm 3.1$ & $9.6 \pm 3.3$ & $0.9(16.8)$ & $<0.01$ & 0.51 & 0.80 \\
\hline \multicolumn{11}{|l|}{ Lumen area } \\
\hline Maximal & $15.5 \pm 9.2$ & $16.0 \pm 8.9$ & $5.1(32.8)$ & 0.18 & $15.6 \pm 7.2$ & $17.6 \pm 6.8$ & $2.0(12.6)$ & 0.01 & 0.99 & 0.09 \\
\hline Mean & $9.4 \pm 5.0$ & $11.4 \pm 5.6$ & $2.0(21.3)$ & $<0.01$ & $9.7 \pm 4.1$ & $11.1 \pm 3.5$ & $1.3(13.4)$ & $<0.01$ & 0.81 & 0.16 \\
\hline Minimal & $5.8 \pm 3.2$ & $7.6 \pm 3.5$ & $1.8(31.0)$ & $<0.01$ & $5.9 \pm 2.9$ & $7.1 \pm 2.2$ & $1.2(20.7)$ & $<0.01$ & 0.80 & 0.25 \\
\hline \multicolumn{11}{|l|}{ Stent area } \\
\hline Maximal & $9.9 \pm 3.7$ & $13.8 \pm 4.8$ & $4.0(40.0)$ & $<0.01$ & $10.3 \pm 3.1$ & $12.9 \pm 3.1$ & $2.6(25.2)$ & $<0.01$ & 0.80 & 0.04 \\
\hline Mean & $8.0 \pm 3.1$ & $11.0 \pm 3.8$ & $3.0(37.5)$ & $<0.01$ & $8.4 \pm 2.7$ & $10.0 \pm 2.4$ & $1.6(19.0)$ & $<0.01$ & 0.89 & $<0.01$ \\
\hline Minimal & $6.4 \pm 2.8$ & $8.3 \pm 3.5$ & $1.9(29.7)$ & $<0.01$ & $6.6 \pm 2.3$ & $7.4 \pm 2.1$ & $0.8(12.1)$ & 0.01 & 0.73 & $<0.01$ \\
\hline Stent expansion & $0.75 \pm 0.31$ & $0.88 \pm 0.25$ & $0.14(27.1)$ & 0.01 & $0.80 \pm 0.24$ & $0.82 \pm 0.26$ & $0.01(12.0)$ & 0.92 & 0.36 & 0.05 \\
\hline \multicolumn{11}{|l|}{ Vessel area } \\
\hline Maximal & $25.7 \pm 9.1$ & $28.0 \pm 9.6$ & $2.4(9.3)$ & $<0.01$ & $26.9 \pm 7.7$ & $29.4 \pm 7.4$ & $2.5(9.3)$ & $<0.01$ & 0.84 & 0.88 \\
\hline Mean & $19.3 \pm 5.7$ & $21.4 \pm 6.4$ & $2.08(10.8)$ & $<0.01$ & $20.0 \pm 4.9$ & $21.5 \pm 5.2$ & $1.5(7.5)$ & $<0.01$ & 0.83 & 0.29 \\
\hline Minimal & $14.3 \pm 5.1$ & $15.3 \pm 5.3$ & $1.0(6.9)$ & 0.01 & $14.7 \pm 4.4$ & $14.2 \pm 4.2$ & $-0.5(-3.5)$ & 0.25 & 0.84 & 0.01 \\
\hline \multicolumn{11}{|l|}{ Malapposition area* } \\
\hline Maximal & 6.9 (4.1 to 11.7 ) & $1.2(0$ to 10.3$)$ & $-4.4(-46.8)$ & $<0.01$ & 7.1 (4.3 to 11.6) & 7.52 (5.4 to 12.7) & $-1.4(-14.3)$ & 0.21 & 0.59 & $<0.01$ \\
\hline Mean & 3.3 (2.0 to 5.2$)$ & 0.49 (0 to 3.6$)$ & $-2.1(-50.0)$ & $<0.01$ & $3.3(2.0$ to 5.1$)$ & 3.7 (1.6 to 5.2) & $-1.3(-27.1)$ & 0.12 & 0.66 & 0.10 \\
\hline Maximal neointimal area & 1.6 (1.0 to 2.6) & 2.5 (1.7 to 3.4$)$ & $0.7(35.5)$ & $<0.01$ & $1.7(0.9$ to 3.1$)$ & 2.1 (1.5 to 2.7) & $0.0(-1.8)$ & 0.94 & 0.94 & 0.17 \\
\hline \multicolumn{11}{|l|}{ Volumes $\left(\mathrm{mm}^{3} \pm \mathrm{SD}\right)$} \\
\hline Lumen volume & $253.1 \pm 196.7$ & $294.3 \pm 214.5$ & $41.2(16.3)$ & $<0.001$ & $237.0 \pm 152.3$ & $392.6 \pm 217.9$ & $155.6(65.7)$ & $<0.001$ & 0.83 & $<0.01$ \\
\hline Stent volume & $207.2 \pm 117.2$ & $279.3 \pm 156.5$ & $72.1(35.0)$ & $<0.001$ & $201.1 \pm 101.0$ & $352.7 \pm 161.9$ & $151.6(43.0)$ & $<0.001$ & 0.45 & $<0.01$ \\
\hline \begin{tabular}{|l|l} 
Vessel volume \\
\end{tabular} & $502.4 \pm 270.2$ & $538.8 \pm 284.1$ & $36.4(7.2)$ & 0.004 & $482.4 \pm 240.2$ & $762.5 \pm 372.7$ & $280.2(58.1)$ & $<0.001$ & 0.50 & $<0.01$ \\
\hline \multicolumn{11}{|l|}{ Malapposition volume * } \\
\hline $\begin{array}{l}\text { Malap./lumen, } \\
\% \pm \text { SD* }^{*}\end{array}$ & \begin{tabular}{|c|}
$34.6(15.3$ to 90.8$)$ \\
$21.5 \pm 15.7$
\end{tabular} & $\begin{array}{c}1.5(0 \text { to } 57.0) \\
8.4 \pm 12.1\end{array}$ & \begin{tabular}{|c|}
$-33.8(-43.6)$ \\
-13.1 \\
\end{tabular} & \begin{tabular}{|l|}
$<0.001$ \\
$<0.001$
\end{tabular} & \begin{tabular}{|c|}
$52.8(19.5$ to 100.9$)$ \\
$24.9 \pm 17.7$ \\
\end{tabular} & \begin{tabular}{|c|}
$54.9(15.0$ to 117.1$)$ \\
$15.7 \pm 11.4$
\end{tabular} & $\begin{array}{c}-2.1(-2.6) \\
-9.1\end{array}$ & $\begin{array}{l}0.874 \\
0.006\end{array}$ & $\begin{array}{l}0.42 \\
0.35\end{array}$ & $\begin{array}{r}0.03 \\
<0.01 \\
\end{array}$ \\
\hline Neointima volume & $4.6(0$ to 14.1$)$ & $13.0(5.0$ to 19.0$)$ & $5.7(50.0)$ & 0.003 & 3.6 (0.1 to 21.8) & 9.5 (3.7 to 20.2) & $4.8(25.8)$ & 0.381 & 0.51 & 0.41 \\
\hline
\end{tabular}

the group treated with BA had a larger reduction than the group treated with ASI $(13.1 \%$ vs. $9.1 \%$; $<0.01)$. In the group treated with ASI there were no changes in the amount of malapposition volume between pre- and post-intervention (82.3 vs. $80.2 \mathrm{~mm}^{3}$; $\mathrm{p}=0.87)$.

\section{OUTCOMES}

Clinical data were available in 110 patients (95\%) with a median of 2.9 years (IQR 1.9 to 4.6) of follow-up. All patients have completed at least one year of clinical follow-up. A total of 13 patients $(11.8 \%)$ died: $12.1 \%$ treated with BA vs. $11.5 \%$ treated with ASI (HR 0.505, CI: 0.109-2.346; $\mathrm{p}=0.38$ ). Only five deaths
(4.5\%) were from cardiac or unknown causes: $3.4 \%$ vs. $5.8 \%$, respectively (HR 0.818, CI: 0.095-7.035; p=0.86). Target lesion revascularisation was observed in four $(6.8 \%)$ vs. three $(5.8 \%)$ patients treated with BA and with ASI, respectively (HR 0.967, CI: 0.095-9.879; $\mathrm{p}=0.98)$. Recurrent definite or probable ST was documented in five patients $(4.5 \%)$ at a median time of 12 months (IQR 10 to 27$)$ : one $(1.7 \%)$ treated with BA vs. four $(7.7 \%)$ treated with ASI (HR 16.232, CI: 0.640-411.700; $p=0.09$ ). Figure 4 shows the Kaplan-Meier survival curves from cardiac death, target lesion revascularisation and recurrent definite or probable ST. Table 4 shows the clinical, angiographic and procedural characteristics of patients with recurrent ST. 

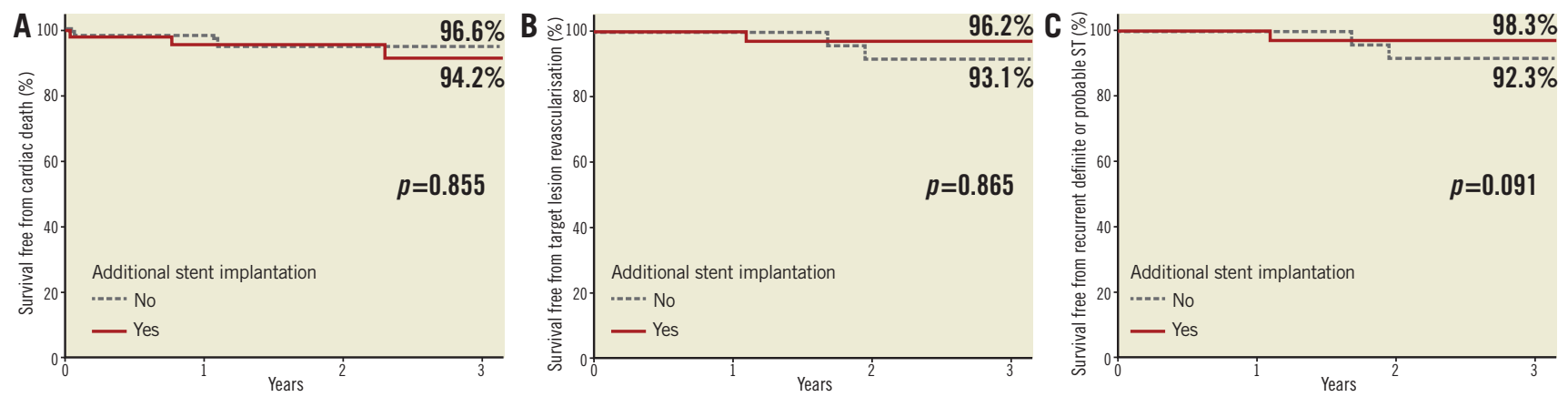

Figure 4. Cardiac outcomes between patients treated with balloon angioplasty vs. additional stent implantation. Survival curves of cardiac death (A), target lesion revascularisation (B) and recurrent stent thrombosis (C).

Table 4. Cases with recurrent stent thrombosis.

\begin{tabular}{|c|c|c|c|c|c|c|}
\hline \multirow{2}{*}{\multicolumn{2}{|c|}{ Demographic characteristics }} & Case 1 & Case 2 & Case 3 & Case 4 & Case 5 \\
\hline & & Man 65 years & Man 56 years & Man 44 years & Man 50 years & Man 39 years \\
\hline \multirow{4}{*}{$\begin{array}{l}\text { Cardiovascular risk } \\
\text { factors }\end{array}$} & Smoking & Ex-smoker & Smoker & Smoker & Smoker & Smoker \\
\hline & Diabetes mellitus & Yes (insulin) & No & No & № & № \\
\hline & Chronic renal failure & Yes (dialysis) & No & No & No & No \\
\hline & Ejection fraction & $41 \%$ & $55 \%$ & $51 \%$ & $60 \%$ & $55 \%$ \\
\hline \multirow{6}{*}{$\begin{array}{l}\text { Characteristics at } \\
\text { the first ST }\end{array}$} & Localisation & Mid-RCA & Distal-RCA & Mid-RCA & Distal-RCA & Proximal-RCA \\
\hline & Type of stent & Everolimus DES & BMS & Zotarolimus DES & BMS & Paclitaxel DES \\
\hline & Sizexlength & $3.5 \times 15 \mathrm{~mm}$ & $4.0 \times 23 \mathrm{~mm}$ & $3.0 \times 30 \mathrm{~mm}$ & $3.5 \times 22 \mathrm{~mm}$ & $3.5 \times 24 \mathrm{~mm}$ \\
\hline & Time to first thrombosis & 52 days & 17 months & 14 months & 5 months & 13 months \\
\hline & Antiplatelet treatment & AAS+Clopidogrel & AAS & AAS & None (stopped 20 days ago) & AAS \\
\hline & Clinical presentation & STEMI (primary PCl) & Non-STEMI & STEMI & STEMI & STEMI \\
\hline \multirow{4}{*}{$\begin{array}{l}\text { IVUS findings of first } \\
\text { ST }\end{array}$} & ISA & Yes $(<2 \mathrm{~mm})$ & Yes (12 mm) & Yes $(30 \mathrm{~mm})$ & Yes $(15 \mathrm{~mm})$ & Yes* $(8 \mathrm{~mm})$ \\
\hline & Aneurysm & No & No & Yes & No & № \\
\hline & Underexpansion & Yes (+++ calcification) & No & No & No & No \\
\hline & Neoatherosclerosis & No & No & No & № & № \\
\hline \multirow{6}{*}{$\begin{array}{l}\text { Treatment of the } \\
\text { first ST }\end{array}$} & Thromboaspiration & No & Yes & Yes & Yes & No \\
\hline & Glycoprotein IIb/IIla inhibitors & No & No & Yes & Yes (post stent) & Yes \\
\hline & Additional stent implantation & Yes & Yes & Yes & Yes & № \\
\hline & Stent type & Paclitaxel DES & BMS & 4 BMS & Zotarolimus DES & № \\
\hline & Procedural complications & No & Yes (haematoma) & No & Yes (embolisation) & № \\
\hline & Final TIMl flow & 3 & 3 & 3 & 2 & 3 \\
\hline \multirow{4}{*}{$\begin{array}{l}\text { IVUS findings after } \\
\text { PCl of first ST }\end{array}$} & ISA & No & Yes $(8 \mathrm{~mm})$ & Yes (49 mm) & Yes (25 mm) & Yes* $(2 \mathrm{~mm})$ \\
\hline & Aneurysm & No & No & Yes & No & No \\
\hline & Underexpansion & No & No & No & № & № \\
\hline & Probable neoatherosclerosis & No & No & No & No & № \\
\hline \multirow{4}{*}{$\begin{array}{l}\text { Characteristics at } \\
\text { the second ST }\end{array}$} & Time to first thrombosis & 2.4 years & 13 months & 3.5 years & 8 months & 10 months \\
\hline & Antiplatelet treatment & AAS & AAS+Prasugrel & AAS & AAS+Clopidogrel & AAS+Clopidogrel \\
\hline & Clinical presentation & Sudden death & Non-STEMI & Non-STEMI & STEMI & STEMI \\
\hline & Treatment & - & Balloon angioplasty & Additional stent & Balloon angioplasty & Additional stent \\
\hline
\end{tabular}




\section{Discussion}

The major findings of this study are the following. 1) According to the IVUS images, ISA and stent underexpansion are the most frequent findings observed in patients with late ST (>75\%). 2) Treatment of late ST with BA is associated with fewer patients with persistent ISA, larger expansion of the stent sizes and with greater reduction of the malapposition volume compared to patients treated with ASI. 3) At two years, patients with late ST treated with PCI and imaged with IVUS show favourable outcomes. However, patients treated with BA have a non-significant numeric difference of lower recurrent ST compared to patients treated with ASI.

Non-optimal criteria of stent implantation are commonly observed immediately after stent implantation as assessed by IVUS. Stent malapposition and stent underexpansion are observed in around $10 \%$ and $40 \%$ of patients, respectively ${ }^{18,19}$. Although such IVUS findings are observed in a larger proportion in patients with late $\mathrm{ST}^{20,21}$, several randomised studies comparing angiographically guided vs. IVUS-guided procedures have found no or a mild relationship between these "IVUS findings" and clinical events ${ }^{22}$.

According to pathologic studies, the most important cause of late ST is lack of strut coverage ${ }^{9,23}$. Stents with a ratio of uncovered to total stent struts per section (RUTSS) $>30 \%$ had a higher risk of thrombosis ${ }^{9}$. Pathologic and optical coherence tomography (OCT) studies have also shown that uncovered struts are often malapposed struts ${ }^{24,25}$. ISA has been related to late ST by different mechanisms. First, allergic reactions to the stent components have been associated with positive vessel remodelling, detaching the stent from the vessel wall and causing late acquired stent malapposition ${ }^{15}$. Second, lack of apposition immediately after stent implantation hampers the healing process and increases the likelihood that some of the malapposed struts will never be covered with neointima ${ }^{24,25}$. This process is referred to as persistent ISA and is the most plausible mechanism of malapposition in stents implanted during STEMI. In our study, $>50 \%$ of patients with ST had stents implanted during STEMI at the index procedure. Accordingly, $73 \%$ of patients with STEMI at the index procedure had ISA at the ST procedure compared with $60 \%$ of patients with other clinical indications at the index procedure $(\mathrm{p}=0.12)$.

Stent restenosis has been reported as another important cause of ST. However, the pathophysiologic mechanisms of ST thrombosis in patients with excessive neointima are still uncertain. The disruption of vulnerable neoatherosclerotic plaques has been identified as one potential mechanism of stent thrombosis in patients with excessive neointima. Kang et al observed neoatherosclerosis or neointimal disruptions in $70 \%$ of 33 patients with late ST imaged with $\mathrm{OCT}^{26}$. IVUS assessment of neoatherosclerosis is challenging and has no clear definition. The present study included 25 patients with IVUS criteria of restenosis $(21.4 \%)$. The IVUS images of those patients showed that 20 patients could have neoatherosclerotic plaques according to the definition of the present study. However, it is possible that a considerable number of neoatherosclerotic plaques were unseen because of the low axial resolution of the IVUS images.
Finally, stent thrombosis can be caused by insufficient antiplatelet therapy ${ }^{10}$. However, most of the patients with very late ST ( $>1$ year) have no indications for dual antiplatelet therapy and are considered for single antiplatelet treatment according to the current guidelines ${ }^{12}$.

According to previous studies, it is possible that IVUS imaging would reduce the number of stents used during treatment of late ST. Waldo et al performed IVUS in $27 \%$ of 205 patients with early or late ST, and all cases were treated with ASI $(100 \%)^{11}$. In the RESTART registry, $57 \%$ of 611 patients with sirolimus DES late ST were imaged with IVUS and only $40 \%$ of patients were treated with $\mathrm{ASI}^{3}$. In the present study, $>50 \%$ of patients with ST were studied with intravascular imaging techniques and $46 \%$ of patients were treated with ASI. The use of ASI can also be affected by the presence of stent restenosis. In our study, $60 \%$ of patients with restenosis were treated with ASI. Most of the patients with late ST and restenosis (68\%) had been treated with BMS at the index procedure. Although drug-eluting balloons (DEB) are recommended for treatment of stent restenosis ${ }^{12}$, only two patients in the nonASI group were treated with DEB at the time of the ST. It is possible that the operators would have preferred the use of ASI instead of BA or BA with DEB due to the potential risk of disrupted neoatherosclerotic plaques as the cause of ST. Finally, ASI can also be necessary in cases of persistent thrombus with TIMI flow $<3$ or in cases with periprocedural complications.

Large registries of patients with late ST have shown worse cardiac outcomes in patients treated with ASI as compared to patients treated without $\mathrm{ASI}^{2,5}$. According to the results of the present study, it is possible that patients treated with ASI still had a large malapposition volume and this may increase the risk of recurrent ST. Although both groups were treated using a similar balloon-toartery ratio, the stent segment of the patients treated with ASI was lengthened without the malapposition length and volume being reduced. In contrast, patients treated without ASI had a larger reduction of malapposition volume and, in most cases, there was a complete resolution of the malapposed segment. Therefore, BA assisted by IVUS guidance should be the first intention to treat in cases with ST caused by stent malapposition and/or underexpansion. In these cases, optimisation of antiplatelet treatment with novel antiplatelet drugs would also be advisable. Furthermore, the use of DEB should be considered in cases with IVUS evidence of neointimal proliferation.

\section{Limitations}

This study was observational. All comparisons performed in this study must be considered with caution and are only hypothesisgenerating. The treatment strategies of ST were probably influenced by procedural characteristics that forced the operators to use one of the two strategies. Moreover, no pre-specified criteria to implant ASI or not were used, and all decisions were left to the operator's discretion. The second limitation of the study is that IVUS was only performed in around $50 \%$ of patients presenting to our institutions with definite late ST. However, this percentage 
is one of the highest rates of IVUS-guided procedures for ST treatment according to previous studies. Finally, IVUS is unable to assess the main cause of late stent thrombosis, namely lack of strut coverage.

\section{Conclusions}

The majority of cases with late ST had stent malapposition and stent underexpansion as assessed by IVUS. Patients treated with BA without ASI presented with a larger reduction or correction of the malapposition and underexpansion compared to patients treated with ASI. Moreover, patients treated with BA had similar outcomes to patients treated with ASI, with a non-significant numeric difference of lower risk of recurrent ST. Although further investigations are required, BA without ASI seems to be safe and effective for the treatment of definite late ST.

\section{Impact on daily practice}

Most of the patients suffering from late stent thrombosis (ST) have incomplete stent apposition (ISA) and/or stent underexpansion as assessed by intravascular ultrasound (IVUS). Treatment of late ST with balloon angioplasty (BA) is associated with greater resolution of ISA and stent underexpansion compared to patients treated with additional stent implantation as assessed by IVUS. It is associated with favourable outcomes and a trend towards a lower risk of recurrent thrombosis. The take-home message of this study is that IVUS guidance for treatment of late stent thrombosis without additional stent implantation is safe, effective and can reduce the risk of recurrent thrombosis.

\section{Conflict of interest statement}

The authors have no conflict of interest to declare.

\section{References}

1. Cutlip DE, Windecker S, Mehran R, Boam A, Cohen DJ, van Es GA, Steg PG, Morel MA, Mauri L, Vranckx P, McFadden E, Lansky A, Hamon M, Krucoff MW, Serruys PW; Academic Research Consortium. Clinical end points in coronary stent trials: a case for standardized definitions. Circulation. 2007;115:2344-51.

2. van Werkum JW, Heestermans AA, de Korte FI, Kelder JC, Suttorp MJ, Rensing BJ, Zwart B, Brueren BR, Koolen JJ, Dambrink JH, van't Hof AW, Verheugt FW, ten Berg JM. Longterm clinical outcome after a first angiographically confirmed coronary stent thrombosis: an analysis of 431 cases. Circulation. 2009;119:828-34.

3. Kimura T, Morimoto T, Kozuma K, Honda Y, Kume T, Aizawa T, Mitsudo K, Miyazaki S, Yamaguchi T, Hiyoshi E, Nishimura E, Isshiki T; RESTART Investigators. Comparisons of baseline demographics, clinical presentation, and long-term outcome among patients with early, late, and very late stent thrombosis of sirolimus-eluting stents: Observations from the Registry of Stent
Thrombosis for Review and Reevaluation (RESTART). Circulation. 2010;122:52-61.

4. Raber L, Magro M, Stefanini GG, Kalesan B, van Domburg RT, Onuma Y, Wenaweser P, Daemen J, Meier B, Jüni P, Serruys PW, Windecker S. Very late coronary stent thrombosis of a newer-generation everolimus-eluting stent compared with earlygeneration drug-eluting stents: a prospective cohort study. Circulation. 2012;125:1110-21.

5. de la Torre-Hernandez JM, Alfonso F, Hernandez F, Elizaga J, Sanmartin M, Pinar E, Lozano I, Vazquez JM, Botas J, Perez de Prado A, Hernandez JM, Sanchis J, Nodar JM, Gomez-Jaume A, Larman M, Diarte JA, Rodriguez-Collado J, Rumoroso JR, LopezMinguez JR, Mauri J; ESTROFA Study Group. Drug-eluting stent thrombosis: results from the multicenter Spanish registry ESTROFA (Estudio ESpanol sobre TROmbosis de stents FArmacoactivos). J Am Coll Cardiol. 2008;51:986-90.

6. de la Torre Hernandez JM, Alfonso F, Gimeno F, Diarte JA, Lopez-Palop R, Perez de Prado A, Rivero F, Sanchis J, Larman M, Diaz JF, Elizaga J, Moreiras JM, Gomez Jaume A, Hernandez JM, Mauri J, Recalde AS, Bullones JA, Rumoroso JR, Del Blanco BG, Baz JA, Bosa F, Botas J, Hernandez F; ESTROFA-2 Study Group. Thrombosis of second-generation drug-eluting stents in real practice results from the multicenter Spanish registry ESTROFA-2 (Estudio Espanol Sobre Trombosis de Stents Farmacoactivos de Segunda Generacion-2). JACC Cardiovasc Interv. 2010;3:911-9.

7. Tada T, Byrne RA, Simunovic I, King LA, Cassese S, Joner M, Fusaro M, Schneider S, Schulz S, Ibrahim T, Ott I, Massberg S, Laugwitz KL, Kastrati A. Risk of stent thrombosis among bare-metal stents, first-generation drug-eluting stents, and second-generation drug-eluting stents: results from a registry of 18,334 patients. JACC Cardiovasc Interv. 2013;6:1267-74.

8. Brodie B, Pokharel Y, Fleishman N, Bensimhon A, Kissling G, Hansen C, Milks S, Cooper M, McAlhany C, Stuckey T. Very late stent thrombosis after primary percutaneous coronary intervention with bare-metal and drug-eluting stents for ST-segment elevation myocardial infarction: a 15-year single-center experience. JACC Cardiovasc Interv. 2011;4:30-8.

9. Finn AV, Joner M, Nakazawa G, Kolodgie F, Newell J, John MC, Gold HK, Virmani R. Pathological correlates of late drug-eluting stent thrombosis: strut coverage as a marker of endothelialization. Circulation. 2007;115:2435-41.

10. Holmes DR Jr, Kereiakes DJ, Garg S, Serruys PW, Dehmer GJ, Ellis SG, Williams DO, Kimura T, Moliterno DJ. Stent thrombosis. J Am Coll Cardiol. 2010;56:1357-65.

11. Waldo SW, Armstrong EJ, Yeo KK, Patel M, Reeves R, Macgregor JS, Low RI, Mahmud E, Rogers JH, Shunk K. Procedural success and long-term outcomes of aspiration thrombectomy for the treatment of stent thrombosis. Catheter Cardiovasc Interv. 2013;82:1048-53.

12. Kolh P, Windecker S. ESC/EACTS myocardial revascularization guidelines 2014. Eur Heart J. 2014;35:3235-6.

13. Garcia Del Blanco B, Rumoroso Cuevas JR, Hernandez Hernandez F, Trillo Nouche R. Spanish cardiac catheterization and 
coronary intervention registry. 22nd official report of the Spanish Society of Cardiology Working Group on Cardiac Catheterization and Interventional Cardiology (1990-2012). Rev Esp Cardiol (Engl Ed). 2013;66:894-904.

14. Mintz GS, Nissen SE, Anderson WD, Bailey SR, Erbel R, Fitzgerald PJ, Pinto FJ, Rosenfield K, Siegel RJ, Tuzcu EM, Yock PG. American College of Cardiology Clinical Expert Consensus Document on Standards for Acquisition, Measurement and Reporting of Intravascular Ultrasound Studies (IVUS). A report of the American College of Cardiology Task Force on Clinical Expert Consensus Documents. J Am Coll Cardiol. 2001;37:1478-92.

15. Cook S, Ladich E, Nakazawa G, Eshtehardi P, Neidhart M, Vogel R, Togni M, Wenaweser P, Billinger M, Seiler C, Gay S, Meier B, Pichler WJ, Jüni P, Virmani R, Windecker S. Correlation of intravascular ultrasound findings with histopathological analysis of thrombus aspirates in patients with very late drug-eluting stent thrombosis. Circulation. 2009;120:391-9.

16. Gomez-Lara J, Teruel L, Homs S, Ferreiro JL, Romaguera R, Roura G, Sanchez-Elvira G, Jara F, Brugaletta S, Gomez-Hospital JA, Cequier A. Lumen enlargement of the coronary segments located distal to chronic total occlusions successfully treated with drug-eluting stents at follow-up. EuroIntervention. 2014;9:1181-8.

17. Gomez-Lara J, Brugaletta S, Diletti R, Gogas BD, Farooq V, Onuma Y, Gobbens P, Van Es GA, Garcia-Garcia HM, Serruys PW. Agreement and reproducibility of gray-scale intravascular ultrasound and optical coherence tomography for the analysis of the bioresorbable vascular scaffold. Catheter Cardiovasc Interv. 2011;79:890-902.

18. Russo RJ, Silva PD, Teirstein PS, Attubato MJ, Davidson CJ, DeFranco AC, Fitzgerald PJ, Goldberg SL, Hermiller JB, Leon MB, Ling FS, Lucisano JE, Schatz RA, Wong SC, Weissman NJ, Zientek DM; AVID Investigators. A randomized controlled trial of angiography versus intravascular ultrasound-directed bare-metal coronary stent placement (the AVID Trial). Circ Cardiovasc Interv. 2009;2:113-23.

19. Tanabe K, Serruys PW, Degertekin M, Grube E, Guagliumi G, Urbaszek W, Bonnier J, Lablanche JM, Siminiak T, Nordrehaug J, Figulla H, Drzewiecki J, Banning A, Hauptmann K, Dudek D, Bruining N, Hamers R, Hoye A, Ligthart JM, Disco C, Koglin J, Russell ME, Colombo A; TAXUS II Study Group. Incomplete stent apposition after implantation of paclitaxel-eluting stents or bare metal stents: insights from the randomized TAXUS II trial. Circulation. 2005;111:900-5.

20. Kosonen P, Vikman S, Jensen LO, Lassen JF, Harnek J, Olivecrona GK, Erglis A, Fossum E, Niemela M, Kervinen K,
Ylitalo A, Pietila M, Aaroe J, Kellerth T, Saunamaki K, Thayssen P, Hellsten L, Thuesen L, Niemela K. Intravascular ultrasound assessed incomplete stent apposition and stent fracture in stent thrombosis after bare metal versus drug-eluting stent treatment the Nordic Intravascular Ultrasound Study (NIVUS). Int $J$ Cardiol. 2012;168:1010-6.

21. Pesarini G, Dandale R, Rigamonti A, Pighi M, Zivelonghi C, Mugnolo A, Ferrero V, Vassanelli C, Ribichini F. Late and very late coronary stent thrombosis: Intravascular ultrasound findings and associations with antiplatelet therapy. Catheter Cardiovasc Interv. 2013;82:1056-65.

22. Parise H, Maehara A, Stone GW, Leon MB, Mintz GS. Meta-analysis of randomized studies comparing intravascular ultrasound versus angiographic guidance of percutaneous coronary intervention in pre-drug-eluting stent era. Am $J$ Cardiol. 2011;107:374-82.

23. Nakazawa G, Finn AV, Joner M, Ladich E, Kutys R, Mont EK, Gold HK, Burke AP, Kolodgie FD, Virmani R. Delayed arterial healing and increased late stent thrombosis at culprit sites after drug-eluting stent placement for acute myocardial infarction patients: an autopsy study. Circulation. 2008;118:1138-45.

24. Gomez-Lara J, Radu M, Brugaletta S, Farooq V, Diletti R, Onuma Y, Windecker S, Thuesen L, McClean D, Koolen J, Whitbourn R, Dudek D, Smits PC, Regar E, Veldhof S, Rapoza R, Ormiston JA, Garcia-Garcia HM, Serruys PW. Serial analysis of the malapposed and uncovered struts of the new generation of everolimus-eluting bioresorbable scaffold with optical coherence tomography. JACC Cardiovasc Interv. 2011;4:992-1001.

25. Gutierrez-Chico JL, Wykrzykowska J, Nuesch E, van Geuns RJ, Koch KT, Koolen JJ, di Mario C, Windecker S, van Es GA, Gobbens P, Jüni P, Regar E, Serruys PW. Vascular tissue reaction to acute malapposition in human coronary arteries: sequential assessment with optical coherence tomography. Circ Cardiovasc Interv. 2012;5:20-9, S1-8.

26. Kang SJ, Lee CW, Song H, Ahn JM, Kim WJ, Lee JY, Park DW, Lee SW, Kim YH, Mintz GS, Park SW, Park SJ. OCT analysis in patients with very late stent thrombosis. JACC Cardiovasc Imaging. 2013;6:695-703.

\section{Supplementary data}

Appendix Table 1. Baseline clinical, angiographic and procedural characteristics of patients with stent thrombosis according to the stent type.

Appendix Table 2. IVUS findings at pre-intervention according to the stent type. 


\section{Supplementary data}

Appendix Table 1. Baseline clinical, angiographic and procedural characteristics of patients with stent thrombosis according to the stent type.

\begin{tabular}{|c|c|c|c|c|c|}
\hline & $\mathrm{n}=112$ lesions & $\begin{array}{l}\text { Bare metal stent } \\
\qquad(n=52)\end{array}$ & $\begin{array}{l}\text { First-generation DES } \\
\qquad(n=41)\end{array}$ & $\begin{array}{l}\text { Second-generation } \\
\text { DES }(n=19)\end{array}$ & $p$-value \\
\hline \multicolumn{6}{|c|}{ Clinical and angiographic characteristics at the time of the stent implantation } \\
\hline \multirow{3}{*}{$\begin{array}{l}\text { Clinical indication, } \\
\mathrm{n}(\%)\end{array}$} & Silent or stable angina & $7(13.5)$ & $12(30.0)$ & $1(5.3)$ & \multirow{3}{*}{$<0.01$} \\
\hline & Non-STEMI ACS & $7(13.5)$ & $14(35.0)$ & $12(63.2)$ & \\
\hline & STEMI ACS & $38(73.1)$ & $14(35.0)$ & $6(31.6)$ & \\
\hline \multirow[t]{5}{*}{ Culprit artery, $\mathrm{n}(\%)$} & LAD & $23(44.2)$ & $29(70.7)$ & $12(63.2)$ & \multirow{5}{*}{0.08} \\
\hline & LCF & $5(9.6)$ & $2(4.9)$ & $3(15.8)$ & \\
\hline & RCA & $24(46.2)$ & $8(19.5)$ & $4(21.1)$ & \\
\hline & Left main & 0 & $1(2.4)$ & 0 & \\
\hline & Vein graft & 0 & $1(2.4)$ & 0 & \\
\hline \multicolumn{2}{|l|}{ Number of stents, $\mathrm{n} \pm \mathrm{SD}$} & $1.4 \pm 0.7$ & $1.1 \pm 0.3$ & $1.4 \pm 1.1$ & 0.04 \\
\hline \multicolumn{2}{|c|}{ Total stent length, $\mathrm{mm} \pm \mathrm{SD}$} & $23 \pm 7.6$ & $26.5 \pm 16.5$ & $30.5 \pm 15.0$ & 0.13 \\
\hline \multicolumn{2}{|c|}{ Minimal stent diameter, $\mathrm{mm} \pm \mathrm{SD}$} & $3.2 \pm 0.5$ & $2.8 \pm 0.3$ & $2.8 \pm 0.3$ & $<0.01$ \\
\hline \multicolumn{6}{|c|}{ Clinical characteristics at the time of the stent thrombosis } \\
\hline \multicolumn{2}{|c|}{ Age (years), median (IQR) } & $66.5 \pm 11.8$ & $60.1 \pm 11.2$ & $62.61 \pm 11.5$ & 0.70 \\
\hline \multicolumn{2}{|c|}{ Men, n (\%) } & $50(96.2)$ & $34(82.9)$ & $19(100)$ & 0.02 \\
\hline \multicolumn{2}{|l|}{ Hypertension, n (\%) } & $36(69.2)$ & $29(70.7)$ & $9(47.4)$ & 0.17 \\
\hline \multicolumn{2}{|c|}{ Hypercholesterolaemia, n (\%) } & $36(69.2)$ & $33(80.5)$ & $14(73.7)$ & 0.47 \\
\hline \multicolumn{2}{|c|}{ Diabetes mellitus, $\mathrm{n}(\%)$} & $12(23.1)$ & $19(46.3)$ & $8(42.1)$ & 0.05 \\
\hline \multirow[t]{3}{*}{ Smoking status, n (\%) } & Never & $10(19.2)$ & $10(24.4)$ & $3(15.8)$ & \multirow{3}{*}{0.09} \\
\hline & Ex-smoker & $27(51.9)$ & $12(29.3)$ & $5(26.3)$ & \\
\hline & Current smoker & $15(28.8)$ & $19(46.3)$ & $11(57.9)$ & \\
\hline \multicolumn{2}{|c|}{ Ejection fraction (\%), mean \pm SD } & $50.8 \pm 8.3$ & $56.2 \pm 11.0$ & $51.2 \pm 12.2$ & 0.04 \\
\hline \multirow{3}{*}{$\begin{array}{l}\text { Antiplatelet treatment, } \\
\mathrm{n}(\%)\end{array}$} & None & $2(3.8)$ & $7(17.1)$ & $3(15.8)$ & \multirow{3}{*}{$<0.01$} \\
\hline & Monotherapy & $44(84.6)$ & $30(73.2)$ & $10(52.6)$ & \\
\hline & Dual antiplatelet treatment & $6(11.6)$ & $4(9.7)$ & $6(31.6)$ & \\
\hline \multicolumn{2}{|c|}{ Time to thrombosis (years), median (IQR) } & $4.0(0.6$ to 7.7$)$ & $4.0(2.4$ to 5.8$)$ & 1.7 (0.8 to 3.7$)$ & 0.02 \\
\hline \multirow{2}{*}{$\begin{array}{l}\text { Clinical presentation, } \\
\mathrm{n}(\%)\end{array}$} & Non-STEMI ACS & $8(15.4)$ & $5(12.2)$ & $5(26.3)$ & \multirow{2}{*}{0.61} \\
\hline & STEMI ACS & $44(84.6)$ & $36(87.8)$ & $14(73.7)$ & \\
\hline \multirow[t]{4}{*}{ Killip class, n (\%) } & I & $44(84.6)$ & $30(73.2)$ & $10(52.6)$ & \multirow{4}{*}{0.22} \\
\hline & II & $6(11.5)$ & $7(17.1)$ & $6(31.6)$ & \\
\hline & III & $1(1.9)$ & $3(7.3)$ & $2(10.5)$ & \\
\hline & IV & $1(1.9)$ & $1(2.4)$ & $1(5.3)$ & \\
\hline Angiographic and pro & dural characteristics at the ti & the stent thrombo & & & \\
\hline TIMI flow pre- & 0 & $42(80.8)$ & $28(68.3)$ & $12(63.2)$ & \\
\hline treatment, n (\%) & 1 & $1(1.9)$ & $4(9.8)$ & $1(5.3)$ & \\
\hline & 2 & $5(9.6)$ & $2(4.9)$ & $1(5.3)$ & 0.24 \\
\hline & 3 & $4(7.7)$ & $7(17.1)$ & $5(26.5)$ & \\
\hline Thromboaspiration, n ( & & $42(80.8)$ & $35(85.4)$ & $12(63.2)$ & 0.13 \\
\hline Glycoprotein IIb/IIla in & oitors, $n(\%)$ & $32(61.5)$ & $29(70.7)$ & $11(57.9)$ & 0.54 \\
\hline ST treatment & Balloon angioplasty & $24(46.2)$ & $24(58.5)$ & $11(57.9)$ & 0 \\
\hline & Additional stent implantation & $28(53.8)$ & $17(41.5)$ & $8(42.1)$ & 0.44 \\
\hline Additional stent type, & BMS & $14(50.0)$ & $10(58.8)$ & $2(25.0)$ & \\
\hline & DES & $14(50.0)$ & $7(41.2)$ & $6(75.0)$ & 0.50 \\
\hline
\end{tabular}


Appendix Table 1. Baseline clinical, angiographic and procedural characteristics of patients with stent thrombosis according to the stent type. (cont'd)

\begin{tabular}{|c|c|c|c|c|c|}
\hline & $\mathrm{n}=112$ lesions & $\begin{array}{c}\text { Bare metal stent } \\
\quad(n=52)\end{array}$ & $\begin{array}{l}\text { First-generation DES } \\
\qquad(n=41)\end{array}$ & $\begin{array}{c}\text { Second-generation } \\
\text { DES }(n=19)\end{array}$ & $p$-value \\
\hline \multicolumn{6}{|c|}{ Angiographic and procedural characteristics at the time of the stent thrombosis } \\
\hline \multicolumn{2}{|c|}{ Post-dilatation, n (\%) } & $11(37.9)$ & $7(35.0)$ & $4(50.0)$ & 0.76 \\
\hline \multicolumn{2}{|c|}{ Largest device nominal diameter, $\mathrm{mm} \pm \mathrm{SD}$} & $3.7 \pm 0.6$ & $3.4 \pm 0.4$ & $3.7 \pm 0.71$ & 0.05 \\
\hline \multicolumn{2}{|c|}{ Largest device estimated diameter, $\mathrm{mm} \pm \mathrm{SD}$} & $3.9 \pm 0.7$ & $3.6 \pm 0.4$ & $4.0 \pm 0.7$ & 0.04 \\
\hline \multicolumn{2}{|c|}{ Balloon-to-artery ratio $\pm \mathrm{SD}^{*}$} & $0.90 \pm 0.19$ & $0.93 \pm 0.25$ & $0.92 \pm 0.23$ & 0.12 \\
\hline \multicolumn{2}{|c|}{ Periprocedural complications, n (\%) } & $4(7.7)$ & 0 & 0 & 0.09 \\
\hline \multirow{4}{*}{$\begin{array}{l}\text { TIMI flow post } \\
\text { treatment, n (\%) }\end{array}$} & 0 & 0 & $1(2.4)$ & 0 & \multirow{4}{*}{0.26} \\
\hline & 1 & 0 & 0 & 0 & \\
\hline & 2 & $3(5.8)$ & 0 & 0 & \\
\hline & 3 & $49(94.2)$ & $40(97.6)$ & $10(100.0)$ & \\
\hline
\end{tabular}

Appendix Table 2. IVUS findings at pre-intervention according to the stent type.

\begin{tabular}{|c|c|c|c|c|}
\hline$n=112$ lesions & $\begin{array}{l}\text { Bare metal stent } \\
\qquad(n=52)\end{array}$ & $\begin{array}{l}\text { First-generation DES } \\
\qquad(n=41)\end{array}$ & $\begin{array}{c}\text { Second-generation } \\
\text { DES }(n=19)\end{array}$ & $p$-value \\
\hline None, n (\%) & $1(1.9)$ & $6(14.6)$ & $2(10.5)$ & 0.08 \\
\hline Malapposition, n (\%) & $18(34.6)$ & $19(46.3)$ & $8(42.1)$ & 0.52 \\
\hline Underexpansion, n (\%) & $7(13.5)$ & $7(17.1)$ & 0 & 0.17 \\
\hline Restenosis, n (\%) & $8(15.4)$ & $2(4.9)$ & $2(10.5)$ & 0.27 \\
\hline Malapposition+aneurysm & $7(13.5)$ & $3(7.3)$ & $1(5.3)$ & 0.48 \\
\hline Malapposition+underexpansion, n (\%) & $4(7.7)$ & $2(4.9)$ & $5(26.3)$ & 0.03 \\
\hline Underexpansion+restenosis, n (\%) & $1(1.9)$ & $2(4.9)$ & 0 & 0.50 \\
\hline Malapposition+restenosis, n (\%) & $3(5.8)$ & 0 & 0 & 0.17 \\
\hline Other combinations, n (\%) & $3(5.8)$ & 0 & $1(5.3)$ & 0.31 \\
\hline
\end{tabular}

